\title{
A Fresh Look at the Potential Mechanisms of Progressive Muscle Relaxation Therapy on Depression in Female Patients With Multiple Sclerosis
}

\author{
Seyyedeh Zahra Safi, \\ ${ }^{1}$ Department of Psychology Science, Payame Noor University, Tehran, IR Iran \\ ${ }^{*}$ Corresponding author: Seyyedeh Zahra Safi, Department of Psychology Science, Payame Noor University, Tehran, IR Iran. Tel: +98-9173128868, E-mail: safi5zohreh@gmail.com
}

Received: July 16, 2014; Revised: November 28, 2014; Accepted: January 21, 2015

\begin{abstract}
Background: According to the World Health Organization (WHO) report released in 2000, about 121 million people worldwide suffer from depression. The major depressive disorder (MDD) among multiple sclerosis (MS) patients is one of the most common mood disorders experienced during life. MS patients who simultaneously suffer from depression have reported more severe symptoms of disease and slower adaptation to new conditions, which ultimately increase the cost of treatment.

Objectives: The aim of this study was to assess the effectiveness of progressive muscle relaxation therapy (PMRT) as an adjunctive therapy for reducing level of depression for MS patients.

Materials and Methods: This research had the randomized controlled trial design with pre and posttest. Thirty female patients based on criteria of MS and MDD disease, were selected from the MS Society of Shiraz, Iran. Beck Depression Inventory (BDI-II) was administered at pre and posttest. The participants were randomly allocated to two groups (experimental and control). Twelve sessions of PMRT using Bernstein and Borkovec's method were held for the experimental group. Levin's test, covariance and ANOVA with repeated measures were used for data analysis.

Results: Experimental and control groups were compared before and after treatment. Analysis of covariance showed that seven levels of depression decreased in the experimental group and analysis of repeated measure showed that $49 \%$ of the changes were related to PMRT. Conclusion: According to the results, PMRT is effective in reducing depression. This therapy enables patients to reach relaxation quickly, and thus can cope with depression reactions effectively.
\end{abstract}

Keywords: Depression; Multiple Sclerosis; Muscle Relaxation; Therapy

\section{Background}

Multiple sclerosis (MS) is a disease with a variable course. This disease usually occurs with reversible periods of neurological disability during the third and fourth decades of life and finally, in the sixth or seventh decades of life, this disease becomes an irreversible neurological disorder and permanent disability (1). MS disease is demarcated with inflammation and destruction of white matter of the brain and spinal cord and its replacement with gliotic tissue (2). The etiology of disease this unknown, however, strong evidence for irreversible neurological disability in MS patients indicates that MS disease is an autoimmune disease against central nervous system myelin or neuron degeneration (3).

MS disease has been reported 1.8 times more in females than males and has a profound effect on the personal lives of individuals (4). One million people in the world suffer from MS that is comorbid with psychiatric disorders (5). Cognitive impairment is common in this disease (6). Prevalence of cognitive impairment is about $30.5 \%$, and affects attention, concentration, performance, processing speed and visual perception. Non-somatic symptoms of depression, can predict cognitive performance but it is not clear how depression affects cognitive impairment (7).
On the other hand somatic and non-somatic symptoms of depression predict exacerbation of MS disease (8). Therefore, interaction between medical and psychological variables in MS disease is complex. MS disease usually indicates disparate disease periods (4). The cause and cure are unknown; appearance, removal and even relapsing of symptoms occurs without any warning signs (9) and the onset of the disease may be acute or gradual (10). MS disease may prevent some patients from activities such as, employment, relationships (social, familial and sexual), goals and long-term plans and activities of daily life (9). Therefore these disabilities will challenge people with MS disease, when they are attempting to pursue an active and compatible lifestyle (11). It seems that unpredictable courses of disease activity influence many different fields of the patient's life. Unpredictable periods can make severe feelings of helplessness and depression in patients with MS, while the hopelessness hypothesis states that unpredictable and negative events in the patient's life leads to depression (12). On the other hand, depression is an important predictor of psychological balance of MS patients (13), and studies have confirmed that there are relationships between structural brain le-

Copyright ( ) 2015, Mazandaran University of Medical Sciences. This is an open-access article distributed under the terms of the Creative Commons Attribution-NonCommercial 4.0 International License (http://creativecommons.org/licenses/by-nc/4.0/) which permits copy and redistribute the material just in noncommercial usages, provided the original work is properly cited. 
sions and depression in MS patients (12). There is a possibility that mental health status can change period of MS disease; as Charcot (1879), has discussed, grief and worry might influence onset and exacerbation of disease symptoms. Recent research has provided some evidence regarding the relationship between emotional states and disease activity in MS disease (9).

A variety of studies have confirmed the relationship between structural brain lesions in MS patients and depression (12). Measurement of depression in patients with MS is complicated because some of the symptoms are identical between depression and MS disease (sexual dysfunction, excessive fatigue, cognitive difficulties, psychomotor retardation, mood changes, sleep changes and emotional changes) (14). Patients with MS often hide symptoms of depression and they complain from other symptoms (15). Therefore, treatment plans for depression among MS patients should be treated with an individual and integrated approach (16). Currently there are no definitive treatment for MS disease and medications only reduce relapse rate, prolong remission, limit the onset of new MS lesions, and postpone the development of longterm disability (17). Nowadays the goal of all current and emerging therapeutic strategies for these patients is to return them to a normal life despite of their disease, and several therapies (modafinil, dalfampridine, baclofen, diazepam, gabapentin and opioids) have been used for symptomatic treatment of disability and symptoms, yet they do not improve disease outcome (17).

For managing the symptoms of MS disease and improving or maintaining function and preserving the patient's quality of life, careful clinical monitoring and pharmacologic and non-pharmacologic therapies are recommended (18). Complementary therapies (such as non-pharmacologic therapies) are widely used by MS patients, and progressive muscle relaxation therapy (PMRT) is a form of complementary therapy (19). The relaxation response is a physiological state and incompatible response against stress response (20). Muscle tension is usually associated with stress and anxiety, which are strongly related to depression. In fact, relaxation therapy is comprised of several methods that show patients how to achieve relaxation. Most programs include training special breathing and progressive muscle relaxation procedures (tensionrelease cycles) to reduce physical and mental tension (21).

PMRT was first identified by Jacobson in 1934 as a way for tensing and releasing 16 muscle groups. He stated that the mind and selected muscles work together in an integrated manner. Muscles and body can be relaxed or can be kept away from stress, anxiety and sympathetic activity with mental relaxation (22). Wolpe in 1948 adopted this technique for systematic desensitization therapy. Bernstein and Borkovec in 1973 adopted this technique for stress management in cognitive-behavioral therapy and they developed a shortened and modified form of the PMRT, and currently this form of relaxation therapy is being commonly used. Some of these adjustments are: seven or four muscle groups, relaxation through recall, recall and counting, and counting $(22,23)$.

Previous studies have indicated that depression is prevalent in MS patients and affects treatment adherence and is associated with neurological damage that results from MS (24-42). Despite the high prevalence of depression in patients with MS and its severe impact on physical and mental health, nowadays there are no completely effective treatments for depression (5). There are significant results indicating the effectiveness of PMRT on the normal human brain and patients with MS and a brain lesion that suffer from depression; for example an investigation of 13 controlled studies on patients without brain lesions revealed that PMRT for depression treatment in different patients (such as, multiple somatoform syndrome, cancer disease, pulmonary disease, cardiac disease, muscular pain, tinnitus disease and night eating syndrome) is better than no treatment, placebo treatment, or other behavioral methods. Also follow-up (after several months) treatments were more effective than the first intervention (43-55). A few studies, have shown that PMRT is effective on quality of life, wellbeing and stress management, and this reduces symptoms such as pain, fatigue, anxiety and depression (9, 55-57).

\section{Objectives}

The aim of this study was to determine whether PMRT could reduce the level of major depressive disorder in female MS patients of the Shiraz Multiple Sclerosis Aid Society.

\section{Materials and Methods}

This study was an applied-experimental research with randomized controlled trial design plus pre and posttests. This study compared two groups; experimental and control, where the first group received PMRT and the second group did not receive any treatment for depression, respectively.

In spring 2010, from the 2800 MS patients of the Shiraz Multiple Sclerosis Aid Society (SHMSAS), 30 depressed female volunteers participated in relaxation therapy. They answered the questionnaire before the intervention. Criteria of MS and major depressive disorder (MDD) disease from minimal to severe were confirmed by the SHMSAS and BDI-II, and after the pretest they were randomly divided to two groups. The experimental group received standard care for MS disease plus 60 minutes of psychological intervention (PMRT) for each session (twice a week for six weeks) while the control group received only standard care for MS disease, and at completion the two groups cooperated again in the posttest.

Ethical considerations and rights were applied for this research (respect of the basic rights of patients such as, privacy, cultural and social values, freedom of choice and honesty about characteristics of therapy and therapist's competence). The cases of the two groups were matched 
in terms of age, marital status, education and income. Selection criteria for inclusion and exclusion were as follows: 1- Agreement of the individual to participate in the research, 2- at least literacy for reading and writing, 3physical ability to participate in therapy sessions, 4- doing homework daily at home, 5- filling notes every week, 6- not having any psychotic disorder, bipolar disorder (depressive episode) and major depressive disorder with psychotic features.

There was no drop out of patients during the intervention, and after treatment, patients of both groups answered the questionnaire for the second time.

\subsection{Tools}

In this study progressive muscle relaxation, method of Bernstein et al. (22), and also the Beck Depression Questionnaire (Beck, Epstein, Brown and Steer, 1988) were used, with the reliability and validity examined for the Iranian population (the validity of questionnaire was confirmed by the positive correlation between BDI-II and brief symptom inventory (BSI)); Pearson's coefficient was 0.87 , and factor analysis (to determine underlying relationships between variables) showed three factors: physical, cognitive and affective. The reliability of the questionnaire using the method of internal consistency was 0.91 (Cronbach's alpha coefficient) (58).

The BDI-II was developed based on the criteria of diagnostic and statistical manual of mental disorders, fourth edition (DSM-IV) for diagnosing depressive disorders (59). The BDI-II is a brief scale that is acceptable for researchers in clinical observations and patient description. As a screening instrument, validity and capability of case-finding for this questionnaire has been proved. It is a subjective paper and pencil questionnaire with ordinal scale for depression as a qualitative variable. In addition cut-off points 0-13, 14-19, 20-28 and 29-63 indicate minimal, mild, moderate and severe depression (59).

\subsection{Validity and Reliability}

Content validity of the questionnaire was improved by rewording and adding items to assess DSM-IV criteria for depression. Also validity was confirmed by the positive correlation between Hamilton Depression Rating Scale, Edinburg Postnatal Depression Scale and the Hospital Anxiety, and Depression Scale-Depression, with a Pearson $r$ of 0.62 to 0.81 . The reliability of the questionnaire using retest coefficients and internal consistency was 0.93 (by Pearson's correlation) and 0.91 (by Cronbach's alpha coefficient) (59).

\subsection{The Content of Therapy Sessions}

The best way for muscle relaxation is for muscles to be contracted as much as possible, and then be relaxed suddenly (tension-release sequence). The released force from the treatment increases the excitability threshold. The released force is a big step toward deeper relaxation and patients can understand the feeling of tension and relaxation of muscles (comparative judgment). Relaxation therapy was divided to two parts:

1- The first six sessions: special breathing plus contraction and relaxation of muscles, with gradual reduction of the number of muscles involved.

2- The second six sessions: special breathing plus contraction and relaxation of mind (20).

\subsection{Data Analysis Method}

To eliminate uncontrolled variables (the effect of pretest on the post-test) analysis of covariance was used (analysis of covariance eliminates the covariate variable). Also Levine Test was used for revealing the difference between the two groups. The experimental design of this study included the independent (PMRT) and dependent variable (depression), while analysis of variance used the method of repeated measures.

\section{Results}

Thirty patients participated in this study that were randomly divided in two groups (experimental and control). Tables 1 and 2 were designed for a glimpse to background variables and descriptive statistics of depression.

Table 1 reveals that most patients in this study were in their third decade of life and most of them had a bachelor's degree of education and single patients were more than others while half of them were sick for more than five years and they had experienced symptoms from mild to severe. Table 2 shows that all patients had depression and some of them had severe depression and in the experimental group the mean value indicates mild depression. The research hypothesis stated that, PMRT reduces depression level in MS patients and this hypothesis was checked by Tables 3,4 and 5 .

Table 3, was designed to measure the equality of variances between groups. The results indicate that there was no significant difference in the variance of groups. The purpose of covariance analysis in Table 4 was to eliminate the covariate variable from the dependent variable and to estimate the central indexes. The results showed the mean and standard deviation had increased, by eliminating the impact of pre-test on the post-test and with considering the significance level. After the treatment of the experimental group, a significant reduction in depression variable occurred in seven levels. In Table 5, the impact of the independent variable on depression level was reviewed by analysis of variance with the repeated measures method.

Table 5 showed that the difference between pre-test and post-test was significant $(\mathrm{P}=0.0001)$, indicating a $49 \%$ reduction in depression levels, which means that this reduction had occurred because of the relaxation therapy, with statistical power of $99 \%$. The results of interaction depression and group showed significant increase effect in interaction weeks of treatment and group membership. 


\begin{tabular}{|c|c|c|c|}
\hline & Control & Experimental & Total \\
\hline \multicolumn{4}{|l|}{ Age } \\
\hline Second decade & $4(0.26)$ & $5(0.33)$ & $9(0.60)$ \\
\hline Third decade & $6(0.40)$ & $5(0.33)$ & $11(0.73)$ \\
\hline Fourth decade & $3(0.20)$ & $4(0.26)$ & $7(0.46)$ \\
\hline Fifth decade & $2(0.13)$ & $1(0.60)$ & $3(0.20)$ \\
\hline \multicolumn{4}{|l|}{ Education degree } \\
\hline High school & $4(0.26)$ & $3(0.20)$ & $7(0.23)$ \\
\hline Diploma & $5(0.33)$ & $4(0.26)$ & $9(0.30)$ \\
\hline Bachelor & $6(0.40)$ & $8(0.53)$ & $14(0.46)$ \\
\hline Master & $0(0.00)$ & $0(0.00)$ & $0(0.00)$ \\
\hline \multicolumn{4}{|l|}{ Marital status } \\
\hline Single & $5(0.33)$ & $7(0.46)$ & $12(0.40)$ \\
\hline Married & $6(0.40)$ & $5(0.33)$ & $11(0.36)$ \\
\hline Divorced & $2(0.13)$ & $2(0.13)$ & $4(0.13)$ \\
\hline Widow & $2(0.13)$ & $1(0.60)$ & $3(0.10)$ \\
\hline \multicolumn{4}{|l|}{ Sick precedent } \\
\hline Less than 2 years & $4(0.26)$ & $4(0.26)$ & $8(0.26)$ \\
\hline 2 to 5 Years & $3(0.20)$ & $2(0.13)$ & $5(0.16)$ \\
\hline 5 to 10 Years & $8(0.53)$ & $7(0.46)$ & $15(0.50)$ \\
\hline More than ten years & $0(0.00)$ & $2(0.13)$ & $2(0.60)$ \\
\hline
\end{tabular}

${ }^{\mathrm{a}}$ Data are presented as No. (\%).

Table 2. Descriptive Statistics of Depression Variables

\begin{tabular}{|c|c|c|c|c|c|c|}
\hline & \multicolumn{2}{|c|}{ Control } & \multicolumn{2}{|c|}{ Experimental } & \multicolumn{2}{|c|}{ Total } \\
\hline & Before & After & Before & After & Before & After \\
\hline Mean & 15.86 & 15.93 & 16.20 & 8.40 & 16.03 & 12.16 \\
\hline Median & 13 & 13 & 16 & 5 & 14 & 10 \\
\hline Mode & 11 & 10 & 11 & 5 & 11 & 5 \\
\hline Sum & 238 & 239 & 243 & 126 & 481 & 365 \\
\hline Standard deviation & 9.76 & 9.72 & 9.50 & 9.43 & 9.46 & 10.16 \\
\hline Variance & 95.26 & 94.49 & 90.31 & 88.9 & 89.62 & 103.24 \\
\hline Range & 31 & 31 & 36 & 40 & 37 & 40 \\
\hline Maximum & 34 & 34 & 40 & 40 & 40 & 40 \\
\hline
\end{tabular}

Table 3. Levine Test Results About Equal Variances in the Two Groups

\begin{tabular}{ccccc}
\hline Depression & F & First-Degree of Freedom & Second-Degree of Freedom & Significance Level \\
\hline & 0.463 & 1 & 28 & 0.502 \\
\hline
\end{tabular}

Table 4. The Estimate of Average Depression Variable

\begin{tabular}{lccc}
\hline Experimental Group & Average $^{\mathrm{a}}$ & Number & Meaningful Level \\
\hline Before intervention & $16.08 \pm 1.03$ & 15 & 0.000 \\
After intervention & $8.24 \pm 1.03$ & 15 & 0.000 \\
\hline
\end{tabular}

\footnotetext{
${ }^{\mathrm{a}}$ Data are presented as Mean $\pm \mathrm{SD}$.
} 
Table 5. Results of Variance Analysis with Repeated Measures Method

\begin{tabular}{lcccccc}
\hline Source of Changes & Total Square & Mean Square & F & Significance & Effect & Statistical Power \\
\hline Depression & 224.26 & 224.26 & 27.46 & 0.0001 & 0.49 & 99 \\
Interaction depression and group & 232.06 & 232.06 & 28.41 & 0.0001 & 0.55 & 99 \\
Error & 228.66 & 8.16 & & & & \\
\hline
\end{tabular}

\section{Discussion}

Before treatment of MS disease, patients spent a lot of time trying to control their emotional disorders (like depression). Levels of depression in MS patients were studied by numerous researchers, and the results showed high levels of this complication and that the effects of depression on MS disease exacerbated (24-42). Through identifying and treating the first symptoms of depression, patients can increase their performance within the society.

This study was designed to assess a hypothesis derived from the Gate Theory; this theory states that psychological factors influence physical factors of pain, physical pain and psychological pain. This means that the same way stress and discomfort can exacerbate pain, relief and relaxation can also reduce pain (60). Depression is an overwhelming psychological pain (61), therefore this randomized controlled study was carried out, during 12 sessions, to determine the effects of a relaxation treatment on managing depression in patients with MS. The results showed that there was a significant relationship between treatment and depression. Table 4 shows mild depression in the experimental group and that after intervention there was a reduction of depression; more specifically, the results showed that patients in the experimental group indicated state of depression like normal people. Analysis of variance showed that these changes (49\%) in the experimental group were a result of PMRT. Table 2 shows severe depression in some cases before and after treatment. These cases often hide symptoms of depression or can't recognize between symptoms of depression and MS disease. Also previous treatments (like pharmacologic therapy) for severe depression were continued. These cases took less advantage from this treatment.

The findings of this study showed that the level of depression (first symptoms of depression), was reduced even with short-term treatment, which was for six weeks (safe, inexpensive and effective intervention), and demonstrated the effectiveness of PMRT in reducing depression as an adjunctive and non-pharmacologic treatment (when treatment was used systematically). The findings of this short-term treatment were consistent with the research of Jorm et al. (21). They confirmed that PMRT, as a psychological intervention for depression, is more acceptable than other interventions (relaxation imagery and autogenic training). The findings of Schroder's study (29) demonstrated that both cognitive behavioral therapy (CBT) and PMRT appear to be effective treatments for depression in the normal human brain.

Based on the results of Sutherland et al. (9), Artemiadis et al. (55), Molina-Rueda and Pérez from Spain (56), and Ghafari et al. from Iran (57), potentially, it could be stated that PMRT may provide benefits, during different dimensions of the disease (vitality, fatigue and depression), for the brain affected by MS. However in psychological interventions, therapist training is essential. In fact, the relaxation technique is an acceptable psychological intervention, while it requires less skill and training than other techniques.

Finally, rationale and supporting evidence, and techniques used in PMRT are summarized in this article for intuitive understanding of future researchers about influences of PMRT.

PMRT is an effective treatment for depression in MS patients, although more studies should be done for investigating relaxation therapy as a first-line treatment in a stepped care approach to manage depression in patients with MS. Therefore, to obtain more accurate results, the following recommendations are suggested:

1- It is suggested that future studies, should use more objective clinical and laboratory results.

2-More control of confounding variables can reduce the limitations of the study (such as: sex, social and cultural status and disease progression).

3- Quantity of therapy will be better by using tapes, movies or booklets and long-term treatment.

4- Finally, screening programs are recommended to detect depression in patients with MS to facilitate access to services.

\section{Acknowledgements}

We praise Allah for helping us complete this study and thank all patients and the Shiraz Multiple Sclerosis Aid Society, for their cooperation with this study.

\section{Author's Contributions}

Seyyedeh Zahra Safi conceived and designed the study, collected and interpreted the data, performed the statistical analysis, drafted and revised the manuscript, and approved the final version of the manuscript.

\section{Declaration of Interest}

None.

\section{References}

1. Multiple sclerosis: an immune or neurodegenerative disorder? Trapp BD, Nave KA. Annu Rev Neurosci. 2008;31:247-69.

2. 2. Assessment of serum magnesium, copper, and zinc levels in 
multiple sclerosis (MS) patients. Masoud SA, Fakharian E. IJPBS, 2007;1(2):38

3. 3. Multiple sclerosis. Keegan BM, Noseworthy JH. Annu Rev Med. 2002;53:285-302.

4. 4. Helplessness, self-efficacy, cognitive distortions, and depression in multiple sclerosis and spinal cord injury. Shnek ZM, Foley FW, LaRocca NG, Gordon WA, DeLuca J, Schwartzman HG, et al. Annals of Behavioral Medicine. 1997;19(3):287.

5. 5. Depression in multiple sclerosis: a review of assessment and treatment approaches in adult and pediatric populations. Skokou M, Soubasi E, Gourzis P. Int Scholar Res Notice. 2012;2012

6. 6. Cognitive dysfunction in early multiple sclerosis: altered centrality derived from resting-state functional connectivity using magneto-encephalography. Hardmeier M, Schoonheim MM, Geurts JJ, Hillebrand A, Polman CH, Barkhof F, et al. PLoS One. 2012;7(7):e340

7. 7. Cognitive impairment has a strong relation to nonsomatic symptoms of depression in relapsing-remitting multiple sclerosis. Sundgren M, Maurex L, Wahlin A, Piehl F, Brismar T. Arch Clin Neuropsychol. 2013;28(2):144.

8. 8. Prospective examination of anxiety and depression before and during confirmed and pseudoexacerbations in patients with multiple sclerosis. Burns MN, Nawacki E, Siddique J, Pelletier D Mohr DC. Psychosom Med. 2013;75(1):76.

9. 9. Relaxation and health-related quality of life in multiple sclerosis: the example of autogenic training. Sutherland G, Andersen MB, Morris T. J Behav Med. 2005;28(3):249.

10. 10. Quality of life and multiple sclerosis: Comparison between people with multiple sclerosis and people from the general population. McCabe MP, McKern S. J Clin Psychol Med Set. 2002;9(4):287.

11. 11. Exercise and multiple sclerosis. White LJ, Dressendorfer RH. Sport Med. 2004;34(15):1077.

12. 12. Attributional style and symptoms of depression in person with multiple sclerosis. Kneebone ,I, Dunmore E. Int J Behav Med. 2004;11(2):110.

13. 13. Impact of depression, fatigue and disability on quality of life in Chinese patients with multiple sclerosis. Chen K, Fan Y, Hu R, Yang T, Li K. Stress Health. 2013;29(2):108.

14. 14. The validity of Beck Depression Inventory and Hamilton Rating Scale for Depression items in the assessment of depression among patients with multiple sclerosis. Moran PJ, Mohr DC. J Behav Med. 2005;28(1):35.

15. 15. The co-occurrence of pain and depression in adults with multiple sclerosis. Alschuler KN, Ehde DM, Jensen MP. Rehabil Psychol. 2013;58(2):217.

16. 16. Stress, depression and antidepressant treatment options in patients suffering from multiple sclerosis. Schumann R, Adamaszek M, Sommer N, Kirkby KC. Curr Pharm Des. 2012;18(36):5837.

17. 17. A review of current and emerging therapeutic strategies in multiple sclerosis. Tullman MJ. Am J Manag Care. 2013;19(2 Suppl):S21.

18. 18. Managing the symptoms of multiple sclerosis: a multimodal approach. Crayton HJ, Rossman HS. Clin Ther. 2006;28(4):445.

19. 19. Effectiveness of applying progressive muscle relaxation technique on quality of life of patients with multiple sclerosis Ghafari S, Ahmadi F, Nabavi M, Anoshirvan K, Memarian R, Rafatbakhsh M. J Clin Nurs. 2009;18(15):2171.

20. 20. Psychobiological changes from relaxation response elicitation: long-term practitioners vs. novices. Chang BH, Dusek JA Benson H. Psychosomatics. 2011;52(6):550.

21. 21. Relaxation for depression. Jorm AF, Morgan AJ, Hetrick SE. J Cochrane Database Syst Rev. 2008;8(4)

22. 22. Bernstein D, Borkovec T, Carlson K. Progressive relaxation training: a manual for the helping profession. Mashhad: Ferdowsi University Press; 1992.

23. 23. Progressive muscle relaxation. McCallie MS, Blum CM, Hood CJ. J Human Behav Social Environ. 2006;13(3):51.

24. 24. Effectiveness of psychologically focused group interventions for multiple sclerosis: A review of the experimental literature. Firth N. J Health Psychol. 2013;19(6):789.
25. 25. Depression screening in Black Americans with multiple sclerosis. Stepleman LM, Decker M, Rollock M, Casillas R, Brands T. Psychol Health Med. 2014;19(1):33.

26. 26. Predictive value of health-related quality of life in progression of disability and depression in persons with multiple sclerosis: a 3-year study. Kisic Tepavcevic D, Pekmezovic T, Stojsavljevic N, Kostic J, Dujmovic Basuroski I, Mesaros S, et al. Acta Neurol Belg. 2013;113(4):403.

27. 27. Stress-stimulated volitional coping competencies and depression in multiple sclerosis. Nielsen-Prohl J, Saliger J, Guldenberg V, Breier G, Karbe H. J Psychosom Res. 2013;74(3):221

28. 28. Symptom overlap in anxiety and multiple sclerosis. O Donnchadha S, Burke T, Bramham J, O'Brien MC, Whelan R, Reilly R, et al. Mult Scler. 2013;19(10):1349.

29. 29. Cognitive behavioral therapy versus progressive muscle relaxation training for multiple somatoform symptoms: Results of a randomized controlled trial. Schroder A, Heider J, Zaby A, Gollner R. Cog Thera Res. 2013;37(2):296.

30. 30. Psychiatric symptoms in patients with multiple sclerosis. Sarisoy G, Terzi M, Gumus K, Pazvantoglu O. Gen Hosp Psychiatry. 2013;35(2):134.

31. 31. Prevalence and concurrence of anxiety, depression and fatigue over time in multiple sclerosis. Wood B, van der Mei IA, Ponsonby AL, Pittas F, Quinn S, Dwyer T, et al. Mult Scler. 2013;19(2):217.

32. 32. A large-scale study of anxiety and depression in people with Multiple Sclerosis: a survey via the web portal of the UK MS Register. Jones KH, Ford DV, Jones PA, John A, Middleton RM, LockhartJones H, et al. PLoS One. 2012;7(7):e340

33. 33. Fatigue, depression and sleep disturbances in Iranian patients with multiple sclerosis. Ghajarzadeh M, Sahraian MA Fateh R, Daneshmand A. Acta Med Iran. 2012;50(4):244.

34. 34. Evaluation of the 9-item Patient Health Questionnaire (PHQ9) as an assessment instrument for symptoms of depression in patients with multiple sclerosis. Sjonnesen K, Berzins S, Fiest KM, M. Bulloch AG, Metz LM, Thombs BD, et al. Postgrad Med. 2012;124(5):69.

35. 35. Quality of life and depression in multiple sclerosis patients: longitudinal results of the BetaPlus study. Pozzilli C, Schweikert B, Ecari U, Oentrich W, Bugge JP. J Neurol. 2012;259(11):2319.

36. 36. Type $\mathrm{D}$, anxiety and depression in association with quality of life in patients with Parkinson's disease and patients with multiple sclerosis. Dubayova T, Krokavcova M, Nagyova I, Rosenberger J, Gdovinova Z, Middel B, et al. Qual Life Res. 2013;22(6):1353.

37. 37. The multiple sclerosis depression rating scale. Fiest KM, Patten SB. Expert Rev Neurother. 2012;12(9):1053.

38. 38. Fatigue and its association with sleep disorders, depressive symptoms and anxiety in patients with multiple sclerosis. Labuz-Roszak B, Kubicka-Baczyk K, Pierzchala K, MachowskaMajchrzak A, Skrzypek M. Neurol Neurochir Pol. 2012;46(4):309.

39. 39. Multiple sclerosis relapses and depression. Moore P, Hirst C Harding KE, Clarkson H, Pickersgill TP, Robertson NP. J Psychosom Res. 2012;73(4):272.

40. 40. Longitudinal assessment of anxiety, depression, and fatigue in people with multiple sclerosis. Brown RF, Valpiani EM, Tennant CC, Dunn SM, Sharrock M, Hodgkinson S, et al. Psychol Psychother. 2009;82(Pt 1):41.

41. 41. Is health anxiety a significant problem for individuals with multiple sclerosis? Kehler MD, Hadjistavropoulos HD. J Behav Med. 2009;32(2):150.

42. 42. Fatigue and social impairment in multiple sclerosis: the role of patients' cognitive and behavioral responses to their symptoms. Skerrett TN, Moss-Morris R. J Psychosom Res. 2006;61(5):587.

43. 43. Impact of applied progressive deep muscle relaxation train ing on the level of depression, anxiety and stress among prostate cancer patients: a quasi-experimental study. Isa MR, Moy FM, Abdul Razack AH, Zainuddin ZM, Zainal NZ. Asian Pac J Cancer Prev. 2013;14(4):2237.

44. 44. Treatments for anxiety and depression in patients with chronic obstructive pulmonary disease: a literature review. Cafarella PA, Effing TW, Usmani ZA, Frith PA. Respirology. 2012;17(4):627.

45. 45. Comparison of relaxation training with a cognitive-behav- 
ioural intervention for indicated prevention of depression in university students: a randomized controlled trial. Vazquez FL, Torres A, Blanco V, Diaz O, Otero P, Hermida E. J Psychiatr Res. 2012;46(11):1456.

46. 46. The effects of progressive muscle relaxation and autogenic relaxation on young soccer players' mood states. Hashim HA, Hanafi Ahmad Yusof H. Asian J Sports Med. 2011;2(2):99.

47. 47. Relaxation response and spirituality: Pathways to improve psychological outcomes in cardiac rehabilitation. Chang $\mathrm{BH}$, Casey A, Dusek JA, Benson H. J Psychosom Res. 2010;69(2):93.

48. 48. Effects of progressive muscle relaxation training on anxiety and depression in patients enrolled in an outpatient pulmonary rehabilitation program. Lolak S, Connors GL, Sheridan MJ, Wise TN. Psychother Psychosom. 2008;77(2):119.

49. 49. Relaxation as treatment for chronic musculoskeletal pain-a systematic review of randomised controlled studies. Persson AL, Veenhuizen H, Zachrison L, Gard G. Phys Ther Rev. 2008;13(5):355.

50. 50. Night eating syndrome: effects of brief relaxation training on stress, mood, hunger, and eating patterns. Pawlow LA, O'Neil PM, Malcolm RJ. Int J Obes Relat Metab Disord. 2003;27(8):970.

51. 51. Biofeedback-assisted relaxation therapy in neurocardiogenic syncope: a pilot study. McGrady AV, Kern-Buell C, Bush E, Devonshire R, Claggett AL, Grubb BP. Appl Psychophysiol Biofeedback. 2003;28(3):183.

52. 52. Relaxation and imagery for anxiety and depression control in community patients with advanced cancer. Sloman R. Cancer Nurs. 2002;25(6):432.

53. 53. Impact of a relaxation training on psychometric and immu- nologic parameters in tinnitus sufferers. Weber C, Arck P, Mazurek B, Klapp BF. J Psychosom Res. 2002;52(1):29.

54. 54. The effectiveness of relaxation training in reducing treatment-related symptoms and improving emotional adjustment in acute non-surgical cancer treatment: a meta-analytical review. Luebbert K, Dahme B, Hasenbring M. Psychooncology. 2001;10(6):490.

55. 55. Stress management and multiple sclerosis: a randomized controlled trial. Artemiadis AK, Vervainioti AA, Alexopoulos EC, Rombos A, Anagnostouli MC, Darviri C. Arch Clin Neuropsychol. 2012;27(4):406.

56. 56. Multiple sclerosis and relaxation techniques. Molina-Rueda F, Perez de la C. J Revista Iberoamericana Kinesiology and Physiotherapy. 2009;12(1):28.

57. 57. Effects of applying progressive muscle relaxation technique on depression, anxiety and stress of multiple sclerosis patients in Iran National MS Society. Ghafari S, Ahmadi F, Nabavi M, Memarian R. Pejouhesh. 2008;32(1):45.

58. 58. Psychometric characteristics of BDI-II in patients of MDD. Dabson KE, Mohamadkhani P. J Rehab. 2007;8(29):104.

59. 59. Assessment of depression in medical patients: a systematic review of the utility of the Beck Depression Inventory-II. Wang YP, Gorenstein C. Clinics (Sao Paulo). 2013;68(9):1274.

60. 60. Wilson P. Immediate Relief. Tehran: Peykan Press; 2000.

61. 61. Sadok VA, Kaplan H. Kaplan and Sadock' synopsis of psychiatry: behavioral sciences - clinical psychiatry. Tehran: Shahre Abe Press; 2003. 